\title{
Efficient and Accurate Modeling of Reflectarray Unit Cells Using SVMs
}

\author{
Daniel R. Prado*†, Jesús A. López-Fernández*, Manuel Arrebola* and Fernando Las-Heras* \\ ${ }^{*}$ Group of Signal Theory and Communications, Universidad de Oviedo, Spain \\ \{drprado, jelofer, arrebola, flasheras\}@ @tsc.uniovi.es \\ ${ }^{\dagger}$ Institute of Sensors, Signals and Systems, Heriot-Watt University, Edinburgh, U.K.
}

\begin{abstract}
This paper presents a technique for an efficient and accurate characterization of dual-polarized reflectarray unit cells by means of Support Vector Machines (SVMs) to accelerate the analysis and optimization of this type of antenna. The goal is to accurately obtain the matrix of complex reflection coefficients using geometrical data of the unit cell. To this end, SVMs are employed to obtain surrogate models of those coefficients in an efficient fashion by employing a novel grid search which accelerates by three orders of magnitude the training process with regard to an exhaustive grid search. In addition, the precision of the surrogate model is tested against full-wave simulations assuming local periodicity obtaining a high degree of agreement.
\end{abstract}

Index Terms-Support Vector Machine (SVM), surrogate model, unit cell, reflection coefficient, reflectarray

\section{INTRODUCTION}

A fast and accurate characterization of reflectarray unit cells is necessary to tackle the optimization of very large antennas at an affordable cost, in particular the crosspolar optimization in which the full matrix of complex reflection coefficients is needed to correctly predict the crosspolar far field [1]. To that end, the use of surrogate models by means of Artificial Neural Networks (ANNs) has already been reported [2], [3] obtaining limited results in the cross-coefficients.

In this work, we propose the use of Support Vector Machines (SVMs) to model the reflectarray unit cell. SVMs overcome the ANNs limitation of overfitting and require less training patterns. A strategy to accelerate the SVM training process is presented and comparisons with full wave simulations based on local periodicity of the full matrix of reflection coefficients are presented to assess the error of the surrogate model.

\section{REFLECTARRAY ANALYSIS}

Reflectarrays are classified as planar apertures and thus its far field may be computed from the tangential reflected field on its aperture. This field may be expressed as a function of the incident electric field radiated by the feed as

$$
\vec{E}_{\mathrm{ref}}^{X / Y}\left(x_{m}, y_{n}\right)=\mathbf{R}^{m n} \cdot \vec{E}_{\mathrm{inc}}^{X / Y}\left(x_{m}, y_{n}\right),
$$

where the superscript indicates the antenna polarization, $\left(x_{m}, y_{n}\right)$ are the coordinates of the $(m, n)$ th element and

$$
\mathbf{R}^{m n}=\left(\begin{array}{cc}
\rho_{x x}^{m n} & \rho_{x y}^{m n} \\
\rho_{y x}^{m n} & \rho_{y y}^{m n}
\end{array}\right),
$$

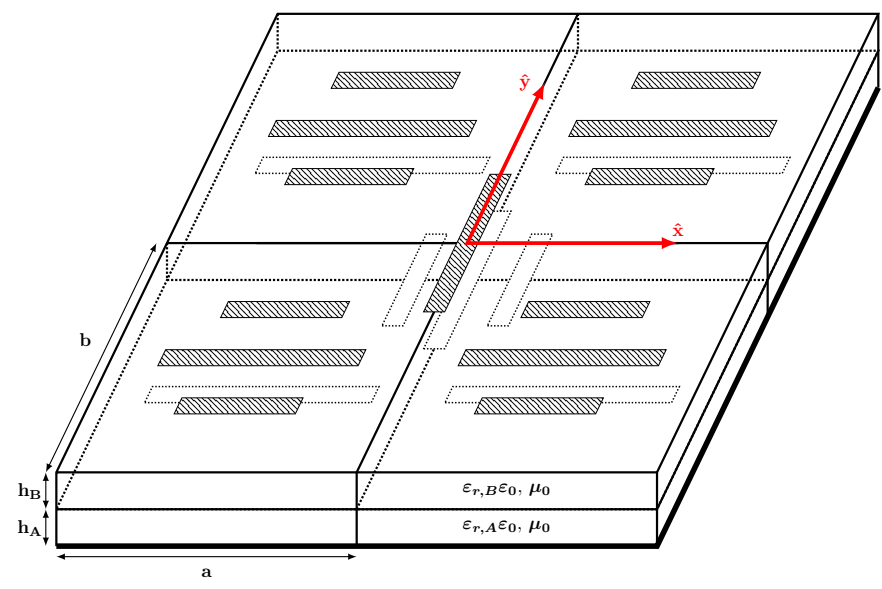

Figure 1. Employed reflectarray unit cell based on parallel dipoles.

is the reflection coefficient matrix which relates both fields at each element. For an accurate computation of the copolar pattern, only the phases of $\rho_{x x}^{m n}$ and $\rho_{y y}^{m n}$ are required. However, a correct prediction of the crosspolar pattern requires the knowledge of the full $\mathbf{R}^{m n}$ matrix, which can only be obtained by relatively slow full-wave computations based on local periodicity [4].

\section{Characterization of Unit Cells with SVM}

The goal of using SVMs is to accelerate the analysis of the reflectarray unit cell while obtaining a high degree of accuracy in the $\mathbf{R}^{m n}$ matrix. The chosen unit cell is shown in Figure 1 and is comprised of two sets of parallel and coplanar dipoles in two layers of metallization, each one controlling one polarization. In this case, $\mathbf{R}^{m n}$ is computed using the spectral domain Method of Moments based on Local Periodicity (MoM-LP) presented in [5]. This unit cell provides plenty of degrees of freedom. Thus, in order to simplify the SVM modeling and increase accuracy, it is proposed to reduce the number of variables. First, the substrate is fixed, choosing the commercially available CuClad 233 with thickness $0.787 \mathrm{~mm}$, $\epsilon_{r}=2.33$ and $\tan \delta=0.0013$. The periodicity is also fixed at $5.84 \mathrm{~mm} \times 5.84 \mathrm{~mm}$, which is approximately half a wavelength at the working frequency, $25.5 \mathrm{GHz}$.

The main factor producing a phase-shift in this unit cell is the dipole lengths. Hence, the separation between dipoles is fixed to $4 \mathrm{~mm}$ and the dipole widths are also fixed to $0.5 \mathrm{~mm}$. 


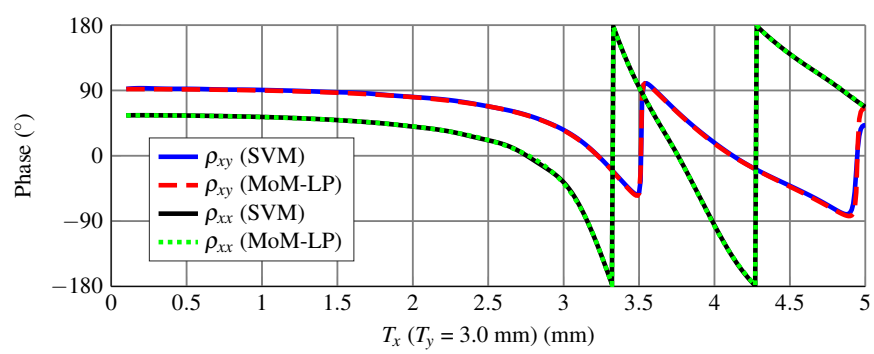

(a)

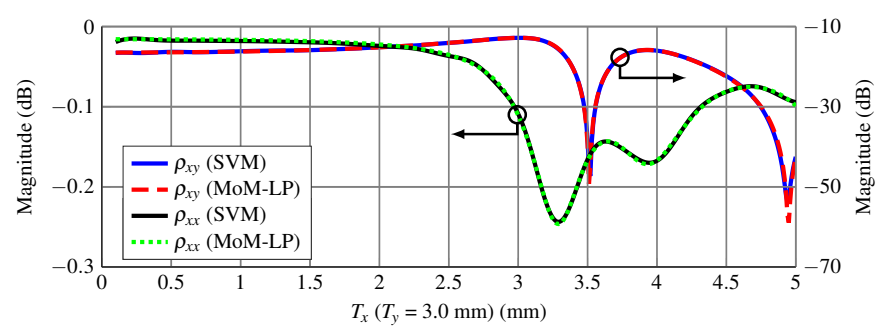

(b)

Figure 2. Comparison of $\rho_{x x}\left(\theta=40^{\circ}, \varphi=0^{\circ}\right)$ and $\rho_{x y}\left(\theta=30^{\circ}, \varphi=\right.$ $60^{\circ}$ ) between SVM and MoM-LP as a function of $T_{x}$ for the cut $T_{y}=3 \mathrm{~mm}$ in (a) phase and (b) magnitude. These two coefficients present the highest error for a direct and cross-coefficient.

In addition, to reduce complexity, one SVM will be trained per angle of incidence $(\theta, \varphi)$ of the impinging plane wave. Finally, the potential degrees of freedom are reduced to only eight dipole lengths. However, to further reduce the model complexity and increase its accuracy, two new variables are defined, $T_{x}$ and $T_{y}$, which will control the dipole lengths of each polarization in such a way that $T_{x}$ controls the lengths of the dipoles oriented in $\hat{x}$ and $T_{y}$ the lengths of the dipoles oriented in $\hat{y}$.

The training of each SVM requires an exhaustive grid search in the hyperparameter space which can be very time consuming. For a Gaussian Kernel with parameters $(C, \gamma)$ [6], it took almost $14 \mathrm{~h}$ to train one single SVM with 1750 training patterns, which provided a high degree of accuracy in the obtained model. Taking into account that hundreds of SVM are needed, it is not practical to perform an exhaustive grid search with so many training patterns. Instead, the following strategy is followed to maintain the accuracy while substantially reducing the training time:

1) Perform an exhaustive grid search using a fraction of the training patterns $(\sim 20 \%)$, identifying the best SVM model.

2) Perform a localized grid search using all training patterns (1750) around the best SVM model obtained in the previous step.

3) If the obtained error is low enough, stop the search. Otherwise, use the Nelder-Mead method to obtain the best model.

With this strategy, the SVM training time was reduced from almost $14 \mathrm{~h}$ to only $65 \mathrm{~s}$, a reduction of three orders of magnitude, while keeping a similar error.

\section{RESUlts}

A number of SVMs were trained for different angles of incidence, and Figure 2 shows the two coefficients (direct and cross-coefficient) with the highest error. The SVM simulations are compared with the full-wave simulation based on local periodicity from which the training samples were obtained. As it can be seen, the agreement is very high, despite being the coefficients with the highest error. For the case of $\rho_{x y}$ the mean absolute error of its phase is $1.17^{\circ}$, while it is $0.39^{\circ}$ for $\rho_{x x}$. In the case of the magnitude, the difference between SVM and MoM-LP is below $-50 \mathrm{~dB}$ for the cross-coefficient $\rho_{x y}$ and below $-70 \mathrm{~dB}$ for the direct coefficient $\rho_{x x}$.

The precision obtained in the modeling of unit cells using SVMs allow to compute the radiation patterns of dualpolarized reflectarrays with an error smaller than $0.6 \%$ for the copolar pattern and $1.4 \%$ for the crosspolar pattern in both polarizations with regard to MoM-LP simulations while achieving a speed up factor larger than 2500, which make the technique very suitable for reflectarray optimization.

\section{CONCLUSION}

A technique for a fast and accurate characterization of reflectarray unit cells using SVMs has been presented. It relies on a series of simplifications to reduce the number of degrees of freedom and a novel grid search to substantially accelerate the training process by a factor of three orders of magnitude while providing a high degree of agreement with MoM-LP simulations, as demonstrated in the simulations of the two reflection coefficients with the highest error.

\section{ACKNOWLEDGMENT}

This work was supported in part by the European Space Agency (ESA) under contract ESTEC/AO/1-7064/12/NL/MH; by the Ministerio de Economía y Competitividad (Spanish Government), under projects TEC2017-86619-R (ARTEINE) and TEC2016-75103-C2-1-R (MYRADA); and by the Gobierno del Principado de Asturias through Programa "Clarín" de Ayudas Postdoctorales / Marie Courie-Cofund under project ACA17-09.

\section{REFERENCES}

[1] D. R. Prado, M. Arrebola, M. R. Pino, R. Florencio, R. R. Boix, J. A Encinar, and F. Las-Heras, "Efficient crosspolar optimization of shapedbeam dual-polarized reflectarrays using full-wave analysis for the antenna element characterization," IEEE Trans. Antennas Propag., vol. 65, no. 2, pp. 623-635, Feb. 2017.

[2] P. Robustillo, J. Zapata, J. A. Encinar, R. Florencio, R. R. Boix, and J. R. Mosig, "Accurate characterization of multi-resonant reflectarray cells by artificial neural networks," in The $8^{\text {th }}$ European Conference on Antennas and Propagation (EUCAP), The Hague, The Netherlands, Apr. 6-11, 2014, pp. 2297-2299.

[3] V. Richard, R. Loison, R. Gillard, H. Legay, and M. Romier, "Loss analysis of a reflectarray cell using ANNs with accurate magnitude prediction," in $11^{\text {th }}$ European Conference on Antennas and Propagation (EuCAP), Paris, France, Mar. 19-24, 2017, pp. 2402-2405.

[4] J. Huang and J. A. Encinar, Reflectarray Antennas. Hoboken, NJ, USA: John Wiley \& Sons, 2008.

[5] R. Florencio, R. R. Boix, and J. A. Encinar, "Enhanced MoM analysis of the scattering by periodic strip gratings in multilayered substrates," IEEE Trans. Antennas Propag., vol. 61, no. 10, pp. 5088-5099, Oct. 2013.

[6] B. Schölkopf and A. J. Smola, Learning with Kernels, 1st ed. Cambridge, Massachusetts: The MIT Press, 2001. 